\title{
La historia de la vejez y su percepción en el CEPE
}

\section{Juan Pablo Vivaldo}

Resumen: El propósito de este artículo es mostrar el impacto y la percepción de la Historia de la vejez durante el Porfiriato (18761911) entre los estudiantes del Centro de Enseñanza para Extranjeros (CEPE). Subrayo que como metodología histórica, la historia de la vejez es una poderosa y novedosa herramienta en la enseñanza de la Historia. Siguiendo esta idea, en primer lugar discuto la historiografía sobre el tema que nos permite entender el alcance de los estudios sobre la historia de la vejez y del envejecimiento; en segundo, presento un breve análisis de la historia de la vejez durante el periodo relacionado con la asistencia a los viejos, la perspectiva médica y las representaciones literarias de la vejez; finalmente muestro algunas experiencias de los estudiantes sobre esta metodología, sus opiniones sobre ella y sobre su propio proceso de envejecimiento.

Palabras clave: Historia de la vejez, Envejecimiento, Porfiriato, CEPE, Estudiantes.

\begin{abstract}
The purpose of this article is to show the impact and the perception of the History of Old Age during the Porfiriato (1876-1911) among the students of the Centro de Enseñanza para Extranjeros (CEPE). I point out that as a historical methodology, History of Old Age is a powerful and new tool in the teaching of History. Following this idea in mind, first of all I discuss the historiography about the subject which allow us to understand the scope of the studies about History Old Age and Ageing in a historical perspective, secondly I present a brief analysis of the History of Old Age during the period related with assistance to the old, the medical perspective and literary representations of Old Age; finally I show different experiences of the students above this methodology, their opinions about it and their own ageing process.
\end{abstract}

Keywords: History of Old Age, Ageing, Porfiriato, CEPE, Students.

En mi experiencia docente en el Centro de Enseñanza para Extranjeros (CEPE) he podido darme cuenta del gusto que despierta entre los estudiantes analizar los procesos históricos que un país ajeno al suyo ha experimentado a lo largo de la diacronía histórica. De forma concreta, en el curso que he impartido durante los últimos años, nos concentramos en el Porfiriato, periodo que se divide en 
los gobiernos del general Porfirio Díaz (1876-1880, 1884-1911) y del también general Manuel González (1880-1884), etapa que debe ser matizada para tener un panorama lo más amplio posible sobre la serie de rupturas y continuidades respecto a los regímenes anteriores.

Para estudiar al Porfiriato he echado mano de una serie de herramientas metodológicas que permiten que el estudiante se involucre y se sienta cómodo en el curso. De esta manera, en ocasiones empleo la historia política para explicar el reconocimiento diplomático que distintos gobiernos extranjeros le otorgaron al mexicano; en otras uso la historia económica cuando nos concentramos en las inversiones que algunos empresarios consolidaron durante el periodo; para adentrarnos en el vasto mundo de las tradiciones mexicanas les presento la historia cultural como una manera de aproximación al ámbito popular; y por supuesto, con frecuencia me apoyo en la historia social puesto que en la Historia sus protagonistas principales son los niños, los adultos $y$, desde luego, los viejos.

El objetivo de esta comunicación es dar a conocer el trabajo que he venido realizando desde la óptica de la historia de la vejez para analizar el Porfiriato. En este texto describo tres elementos que están presentes a lo largo de mi curso (que se ubica en un nivel 4 [Básico] de los ocho que integran el programa de español): la historiografía sobre el tema, una breve síntesis de la historia del envejecimiento durante el periodo y las experiencias de algunos estudiantes que pocas veces (o por primera ocasión) han reflexionado sobre el proceso de envejecimiento desde una perspectiva histórica.

Me parece pertinente mencionar que entenderé como vejez a la etapa del ciclo vital que es definida en distintos contextos históricos mediante un consenso, y por envejecimiento a un proceso biológico por el que cursamos los seres vivos, que es gradual y adaptativo y que es proporcional a la edad y al desgaste acumulado, en un contexto personal, histórico y social que involucra procesos subjetivos.

\section{LA HISTORIOGRAFÍA}

Si hacemos un ejercicio de memoria sobre los textos que hemos leído y que se relacionan con la pobación vieja, seguramente recordaremos algunos títulos y vendrán a nuestra mente novelas, cuentos, leyendas, crónicas o relatos que en algún momento de nuestra vida fueron significativos pero que seguramente abordaron a la vejez tangencialmente sin problematizar lo suficiente el tema. Ahora pensemos sobre los trabajos académicos, las investigaciones profesionales o los estudios científicos que se han interesado en estudiar el proceso de envejecimiento humano, supongo que el número de ellos se verá 
reducido puesto que el tópico es relativamente nuevo si hablamos en términos de la historia de la humanidad. Pero ¿qué pasa cuando ponemos nuestra mirada en los trabajos históricos sobre la vejez? (Sospecho que será más difícil enfocar la mirada).

El registro escrito sobre la historia de la vejez (la historiografía de la vejez) es escasa en comparación con otros grupos sociales y etarios. En otras palabras, es más probable encontrar en bibliotecas o en bases de datos estudios históricos sobre la infancia, la adolescencia, el género, la mujer o las masculinidades que localizar aquellos que hacen referencia a la vejez en cualquier periodo histórico. Esto no quiere decir que no existan. Más bien hace alusión al escaso interés de los historiadores por investigar el envejecimiento y la vejez histórica.

La manufactura de la historia de la vejez (History of Old Age) ha sido en su mayoría extranjera y de países no hispanohablantes. En esta ocasión me centraré exclusivamente en la producción surgida durante la segunda mitad del siglo XX pues considero que es hasta ese momento en donde los estudios de carácter histórico sobre la vejez se vuelven más sistemáticos a tal punto que ya es posible hablar de una especialización en el tema. Uno de esos trabajos lo escribió la filósofa francesa Simone de Bauvoir (1908-1986). En 1970 y a los 62 años de edad, escribió un ensayo titulado La Vejez (La Viellesse) preocupada por sacar a la discusión pública "una especie de secreto vergonzoso del cual es indecente hablar" (Beauvoir, 1983, p. 7). Lo interesante de esta propuesta es que la vejez "no sólo es un hecho biológico, sino un hecho cultural" (Beauvoir, 1983, p. 20), ella entendió el envejecimiento como una construcción histórica que se alimenta de los distintos contextos en que es experimentada. Georges Minois (1946) es otro historiador francés que en sus inicios se interesó en estudiar la vejez histórica. En 1987 publicó su Histoire de la vieillesse de l'Antiquité à la Renaissance, en donde analizó "la función social del anciano en sociedades de tipo tradicional antes de la invasión masiva de la imprenta y de la burocracia del Estado" (Minois, 1987, p.19).

Algunos historiadores de los Estados Unidos, pioneros en la investigación sobre la vejez y el envejecimiento histórico han hecho contribuciones fundamentales al tema. David Hacket Fischer (1977) publicó hace más de 30 años Growing Old in America, como un esfuerzo "para entender el problema del envejecimiento actual a través de su historia" (Hacket, 2015, p. 5); mientras que Robert Garland (1993) se encargó de estudiar la vejez histórica en la Grecia Antigua. En 1992, Thomas R. Cole en The Journey of Life. A Cultural History of Aging in America, aportó desde la metodología de la historia cultural un trabajo en donde se muestra la manera en la que cambiaron las percepciones sobre el ciclo vital desde la Reforma hasta la Primera Guerra Mundial (un periodo aproximado de cuatro siglos) (Cole, 2006). Este último autor junto 
con Mary G. Winkler editaron en 1994 The Oxford Book of Aging. Reflections of the Journey of Life, un texto que recoge una serie de reflexiones históricas de una serie personajes reconocidos en disintas áreas del conocimiento sobre el envejecimiento. Por último, Andrew Achenbaum (1995) se ocupó de analizar el surgimiento de la Gerontología en Estados Unidos como la ciencia encargada de mirar al envejecimiento desde una pespectiva más amplia y no únicamente desde el enfoque médico.

La historiografía inglesa sobre la vejez, y que dicho sea de paso, ha producido los estudios más recientes en el tema, ha sido obra de Pat Thane. En Old Age in English History. Past Experiences, Present Issues, la historiadora hace énfasis en el origen del sistema de pensiones en Inglaterra así como en la idea del trabajo y el retiro dentro de la larga duración histórica (Thane, 2000). Un estudio editado por la misma autora (Thane, 2005) nos ofrece un recorrido desde la Roma y Grecia de la Antigüedad hasta el siglo XX.

Para el caso español Víctor Alba (1992) ha analizado las diversas concepciones sobre la vejez a través de la historia lo que le ayuda a reflexionar desde la actualidad sobre el tema. De manera similar, Francisco García González (2005) aborda el tema desde una perspectiva multidisciplinar enfocando su estudio desde distintos ángulos.

Aunque el tema de la vejez en México no ha encontrado trabajos históricos que lo aborden específicamente, partiendo de otros objetivos algunos historiadores brindan algunas referencias al tema. La primera referencia apareció en el trabajo de Moisés González Navarro (González, 1970). Su importancia radica en que fue de los primeros historiadores en darles visibilidad a los viejos como actores históricos así como en mostrar un esbozo de los asilos, hospicios y montepíos que existieron fuera de la Ciudad de México. Sin embargo, a pesar de que aborda este tema en un apartado intitulado: Niños y viejos, el autor sólo ofreció un dato aislado del asilo de mendigos ubicado dentro del Hospicio Cabañas, Guadalajara, en donde se refirió específicamente a los ancianos. Los trabajos de Silvia Marina Arrom $(2000,2011)$ estudian el Hospicio de Pobres (17741886) y los cambios que sufrió en su población, así como una de las primeras asociaciones de asistencia social en el país que se ocuparon de atender a los "menesterosos". En una serie de novedosos trabajos sobre las instituciones públicas que apoyaron a los indigentes de la Ciudad de México en el periodo 1877-1905, María Dolores Lorenzo, encontró a los ancianos como uno de los grupos atendidos en el Hospicio de Pobres, mismo que posteriormente fue trasladado a una institución privada (Lorenzo, 2011, 2012, 2013). Mario Barbosa (2008) estudió el trabajo en las calles de la ciudad a principios del siglo XX en donde abordó el tema del repudio hacia los pobres, incluyendo a los viejos, brindando una serie de fuentes importantes para la investigación que propongo. 
Por su parte, Elisa Speckman Guerra (2002) y James Alex Garza (2008) al analizar la administración de justicia durante el Porfiriato, ofrecen enfoques y problemas que destacan el papel significativo de los ancianos para entender el entramado social y brindan caminos para acercarse a las fuentes judiciales primarias como una veta para estudiar el tema.

\section{Breve síntesis sobre la historia de La Vejez duRante el Porfiriato}

Un proceso social, político o biológico debe ser estudiado en su complejidad y desde distintas aristas. Es por eso que prefiero hablar de vejeces puesto que el contexto en el que vivieron las personas a finales del siglo XIX y principios del XX suele diferenciar su tipo de realidad. Así, una persona que habitó un ámbito rural envejeció de manera distinta a aquella que lo hizo en un ciudad; no fue lo mismo el proceso de envejecimiento de un individuo que tuvo resueltas sus necesidades económicas a otro para quien su futuro inmediato fue incierto y careció de los recursos para satisfacer sus necesidades básicas; existió una marcada diferencia entre los viejos que construyeron redes sociales de apoyo y aquellos que, por distintas circunstancias, se encontraron aislados en la última parte de su vida... fue una experiencia muy distinta envejecer siendo varón que hacerlo como una mujer.

A diferencia de Europa, en donde desde principios del siglo XIX se comenzó a debatir algunas cuestiones relativas al proceso de envejecimiento humano así como a proyectos relacionados con la vejez, en México ese tipo de reflexiones apareció hasta el último tercio del siglo y fue hasta principios del siguiente en donde se mostraron los primeros atisbos de una asistencia enfocada a los ancianos.

Yo me estoy ocupando de estudiar a la vejez (Ilamémosle porfiriana) en distintos campos: en la asistencia social, en donde los esfuerzos del Estado combinados son los de algunos particulares brindaron auxilio a las personas envejecidas; en el terreno de la medicina que fue pionero en la reflexión sobre el proceso de envejecimiento; y por último, en la literatura, que con la variedad de matices y descripciones de los más reconocidos escritores de la época enriquecieron las representaciones de la vejez a través de la prensa.

\section{LA BENEFICENCIA PÚBLICA}

Resulta primordial analizar el terreno de la asistencia social para ampliar la mirada y darnos cuenta que a partir del triunfo liberal el Estado no monopolizó 
el terreno de la beneficencia, sino que al ser más bien débil tuvo que aceptar que grupos privados (filántropos, laicos) complementaran la ayuda que era otorgada a los sectores menesterosos de la población entre los que destacaron los viejos.

Una de los establecimientos públicos que auxilió a los ancianos fue el Hospicio de Pobres, que desde finales del siglo XVIII los albergó entre sus muros. El ingreso en él podía ser por voluntad propia de la persona o mediante el uso de la fuerza (en el caso en que la autoridad judicial lo juzgara pertinente). De acuerdo con Arrom, el hospicio se diseñó no sólo como refugio para los pobres sino también "como escuela para el adoctrinamiento religioso y el entrenamiento vocacional, obraje donde debían trabajar a cambio de comida y alojamientos, reformatorio y prisión" (Arrom, 2011: 80).

Respecto a la asistencia a los ancianos, mientras que el artículo 40 del Reglamento de la Beneficencia Pública de 1881 estipuló el que el Hospicio de Pobres alojaría a ancianos desvalidos de ambos sexos, Mitchel encontró que el reglamento de éste último, expedido en 1884, declaró que "el único propósito de la institución era el cuidado de niños" (Mitchel, 1997: 106). Esto se debió a un par de factores: el primero tuvo que ver con la convicción de que la ayuda del Estado debía enfocarse en los niños y en los jóvenes, no en los menos infractores o en los ancianos "quienes estaban encasillados en el vicio y en la mendicidad" (Lorenzo, 2012: 212); el segundo fue porque en 1884 se decidió trasladar a los ancianos asilados en el Hospicio de Pobres al Asilo Particular de Mendigos.

El traslado obedeció a las cada vez más tensas relaciones entre los ancianos, las autoridades y algunos otros asilados que redundó en que a éstos se les señalara como un grupo conflictivo. Fue el caso del problema que se reportó a las autoridades del establecimiento el 14 febrero de 1880 cuando se descubrió que al hacer la limpieza del dormitorio de ancianos, debajo de la cama de Bernardino Prado había treinta y nueve pares de zapatos "usados algunos pero en muy buen estado y muy viejos los otros". Según las pesquisas de las autoridades, la razón de que el anciano tuviera el calzado bajo su cama obedecía a que éste, mediante una serie de abusos, obligaba a los niños a vender el calzado. Aunque las autoridades tuvieron conocimiento previo de estas atropellos, fue hasta ese momento en el que contaron con una prueba fehaciente del hecho (AHSS, 1880).

Este tipo de abuso fue uno de los pretextos utilizados por las autoridades del establecimiento para justificar su traslado al Asilo Particular de Mendigos en 1884.

\section{LA BENEFICENCIA PRIVADA}

Los esfuerzos de filántropos, grupos religiosos o laicos fueron un importante complemento para un Estado débil al que le costaba cada vez más hacerse 
cargo de la asistencia a la población desprotegida. El 7 de noviembre de 1899, Porfirio Díaz promulgó la Ley de Instituciones de Beneficencia Privada para el Distrito Federal buscando promover la iniciativa de los particulares en el terreno de la asistencia a los sectores más desprotegidos. La importancia de dicha ley fue que por primera ocasión se plantearon proyectos dirigidos exclusivamente a ancianos. En este apartado presento tres establecimientos privados que obtuvieron su personalidad jurídica durante el Porfiriato y que auxiliaron a un sector de la población envejecida.

Uno de los establecimientos más antiguos que surgieron bajo el cobijo de la Beneficencia Privada fue el asilo "Matías Romero" que fue así nombrado para honrar al diplomático y benefactor de la institución que llevó su nombre. El abogado oaxaqueño nombró como su albacea y ejecutora testamentaria a su hermana Luz Romero de García quien Ilegó a la conclusión de que la ancianidad desvalida debía ser la merecedora del apoyo puesto que "tenemos hospitales para toda clase de enfermedades, colegios y orfanatorios pero que hay pocos asilos de ancianos". De esta manera, Luz Romero decidió "formar un establecimiento en el que los ancianos de ambos sexos encuentren habitación y alimento y se remedien sus necesidades por el resto de sus días" (AJAP, 1900: 41).

Sus estatutos contemplaron que las personas permanecerían en él "durante toda su vida siempre que observen buena conducta [...] y no contravengan los estatutos ni los reglamentos interiores del establecimiento"y aludió al hecho de que los asilados debían trabajar "siempre que puedan hacerlo prudentemente", aunque se estableció que no sería obligatorio (AJAP, 1900: 48). Para ser aceptados en el asilo, las personas debían tener "cuando menos cincuenta años de edad" y era necesario que "dos personas de notorio buen nombre certifiquen que el solicitante es acreedor al socorro". Las personas admitidas serían aquellas pertenecientes a la clase alta o media de la sociedad "que hayan llegado a la miseria y no tengan medio alguno de salir de ella" (AJAP, 1900: 49).

El segundo establecimiento que brindó atención a los ancianos y que vio la luz un 23 de junio de 1879, fue el Asilo Particular para Mendigos. Para su fundador, el tipógrafo Francisco Díaz de León, el establecimiento no sería "asilo de la ociosidad" por lo que tanto los talleres como las escuelas existentes en él, impedirían a toda costa su transmisión. Sus estatutos, aprobados el 21 de enero de 1903, lo definieron como una institución de beneficencia privada con el objetivo de ayudar "a las personas que por su edad avanzada o por enfermedad están impedidos para dedicarse a un trabajo lucrativo, y dar asistencia moral y religiosa a los niños que carezcan de personas obligadas a alimentarlos" (JAP, 1934: 205). Los requisitos necesarios para la admisión fueron: comprobar la necesidad imperiosa de los auxilios, tener buenas costumbres y no padecer enfermedades crónicas que requieran cuidado especial o que sean contagiosas, 
pues el asilo no tiene carácter de hospital o sanatorio" (JAP, 1934:206). En el caso del Asilo Particular para Mendigos, no podríamos hablar estrictamente de un establecimiento financiado por la iniciativa privada dado que el ayuntamiento de la ciudad contribuyó con algunos recursos para su mantenimiento.

La tercera institución de beneficencia privada que brindó apoyo exclusivo para las ancianas, fue el Asilo "Casa Betti", nombrado así por la colimense Isabel Lozano viuda de Betti en memoria de su esposo. En ella fueron admitidas:

las mujeres que adoleciendo de enfermedades crónicas, reputadas incurables, no contagiosas, no puedan [...] procurarse por su trabajo o por otros medios los elementos de subsistencia y de asistencia espiritual y médica que sus dolencias requieran y en general de mujeres de avanzada edad (JAP, 1934:161).

Para ser admitidas en el establecimiento, la persona tenía que solicitar a la administración su ingreso, redactar una solicitud en donde añadiera el nombre de otra persona que en caso de ser necesario se hiciera cargo de la asilada, ser valoradas por el médico para constatar que no tuvieran enfermedades contagiosas o que pudieran perjudicar al resto de las ancianas y por último, sujetarse al reglamento interno del asilo.

Con base en las líneas anteriores la asistencia a los ancianos no la monopolizó una sola entidad, los esfuerzos privados fueron un complemento que se hizo necesario en un Estado cuyos esfuerzos para auxiliar a la población más necesitada cada vez resultaban más limitados.

\section{LA VISIÓN DESDE LA MEDICINA}

Los ojos de la medicina porfiriana estuvieron puestos principalmente en terrenos como la higiene, en el combate a las enfermedades infecciosas o en la prevención de las mismas que se enfocaron en la población infantil y adulta. En este apartado mostraré que al menos hubo un par de personas que se preocuparon por comprender el proceso de envejecimiento así como de estudiar las enfermedades de la vejez.

El combate al envejecimiento ha sido un tema recurrente en la historia de la humanidad. Desde la búsqueda de la fuente de la juventud hasta la fabricación de sustancias químicas, la mira ha sido puesta en impedir que el organismo envejezca haciéndolo más propenso a adquirir distintas enfermedades cuyo fin era inevitablemente la muerte.

Aunque encontrar "la cura" del envejecimiento era una idea generalizada, en el viejo continente comenzaron a circular una serie de reflexiones que comenzaron a dar forma a un conocimiento más especializado que se enfocaría 
en el tratamiento médico de las personas viejas, sector de la población que había sido escasamente tomada en cuenta por la ciencia médica.

No tenemos certeza si es que estas innovadoras ideas llegaron a México procedentes de Europa o si se trató de una aportación del pensamiento médico mexicano, lo cierto es que en las últimas décadas del siglo XIX, aparecieron en dos revistas médicas, La Medicina Científica y la Gaceta Médica de México, algunas publicaciones que aludieron a algunos aspectos del envejecimiento.

En La Medicina Científica aparecieron menos de una decena de artículos firmados por Juan Soler y Roig, en donde el autor mostró su preocupación por entender las patologías que se desencadenaban al envejecer. Por desgracia, aún no he podido localizar información sobre él que permitan conocer su formación profesional, su carrera académica o su interés (personal o académico) por estudiar el tema. Tampoco se ha encontrado en esas publicaciones referencias que remitan a autores europeos, sin embargo, existen algunas semejanzas en cuanto a la terapéutica que éstos últimos propusieron con las aportaciones de Soler y Roig.

Describió lo que llamó el marasmo senil así como las patologías de algunos órganos, brindó una serie de recomendaciones para "vivir dilatados años" que estuvieron vinculados a una modificación de la dieta de los ancianos (recomendó la ingesta de huevos, vegetales y frutas); de sus hábitos personales (entre los que destacaba el baño con frecuencia o dormir ocho horas para "despertarse al rayar el alba") así como de su estilo de vida (evitar asistir a lugar conglomerados en donde se respirara el pernicioso humo del tabaco).

José María Bandera (1832-1910) fue el único médico mexicano de quien hasta este momento se tiene la certeza que reflexionó sobre el envejecimiento humano y que publicó en una revista especializada sus resultados para compartirlos con parte de la comunidad médica de la época.

El doctor Bandera fue un médico oftalmólogo a quien su interés lo llevó más tarde a estudiar las enfermedades mentales. El primer artículo que publicó en donde se refiere al envejecimiento fue a la edad de 70 años. No obstante su curiosidad científica lo llevó durante su vida profesional a explorar otros terrenos, no se puede descartar la posibilidad de que su interés por la vejez haya sido potenciado por su propio proceso de envejecimiento.

Al igual que Soler y Roig, a Bandera también le importó transmitir la idea de modificar estilos de vida y la nutrición de los viejos. Lo que trascendió en el pensamiento del galeno y que, desde mi punto de vista representa uno de sus mayores aportes, fue el hecho de considerar al envejecimiento como un proceso biológico que es producto de los hábitos y costumbres de las personas.

Aunque es cierto que no podemos hablar de la existencia de un discurso médico sobre la vejez puesto que, a diferencia de la infancia, el tratamiento de 
las enfermedades de la personas envejecidas no era considerado primordial para el progreso nacional, sí podemos darnos cuenta que dentro de la comunidad científica existieron distintos intereses que llevaron a Juan Soler y Roig y a José María Bandera a explorar el tema del envejecimiento.

\section{LA REPRESENTACIÓN DE LA VEJEZ EN LA LITERATURA}

Gracias a la palabra impresa logramos transportarnos a otras épocas, lugares y circunstancias que, de otra manera, serían imposibles conocer. Una herramienta para darnos una idea sobre las costumbres, el uso del lenguaje y los adjetivos que fueron empleados para referirse a tal o cual persona, es la literatura. En este sentido, las líneas siguientes buscan dar a conocer algunas representaciones de la vejez a través de los textos de algunos de los escritores más afamados del periodo. Por otro lado, me interesa mostrar que dichas representaciones se fueron modificando de manera simultánea a su propio envejecimiento.

Localizar textos en donde aparezca alguna crítica o experiencia sobre la vejez no es labor sencilla puesto que la mayoría de los temas que los escritores imprimieron a través de sus páginas fueron de diversa índole (las complejas relaciones humanas que involucran toda una gama de sentimientos, la narración del progreso material que se experimentó durante el periodo, la descripción de costumbres, festividades y tradiciones de la sociedad de la época, entre otras). Sin embargo, en los textos de algunos de ellos encontramos o bien elucubraciones sobre la vejez o reflexiones sobre su propia forma de envejecer.

En una parte de la obra de Guillermo Prieto (1818-1897), Laura Méndez de Cuenca (1853-1928), Manuel Gutiérrez Nájera (1859-1895), Luis G. Urbina (18641934) y Ángel de Campo (1868-1908), encontramos una serie representaciones sobre lo que implicó ser viejo y que, como anteriormente se comentó, se vincula con el ciclo de vida de los escritores. En otras palabras, no tuvo el mismo significado que un Guillermo Prieto a la edad de 25 años describiera a una personaje viejo que a los 60 años de edad, así como tampoco podemos comparar la visión sobre la vejez de Ángel de Campo o de Manuel Gutiérrez Nájera (el primero fallecido a los 40 y el segundo a los 34 años) con las experiencias de aquellos que pasaron de esas edades como Laura Méndez de Cuenca o Luis G. Urbina.

Así, mientras un joven Guillermo Prieto se refirió a Canuta Cangarrina, la "vejestoria anfibia" que según él lo recibió al llegar a este mundo, poseedora de una"fisonomía neutra"y siempre gustosa de inmiscuirse en la vida privada de los demás (Prieto, 2005: 206-210), casi a la edad de 60 años plasmó en una poema lo que significaba el amor de un viejo así como la tranquilidad y serenidad con que éstos lo envolvían (Prieto, 2005: 405-407). 
Ángel de Campo y Manuel Gutiérrez Nájera también describieron e incluso se burlaron de las personas envejecidas, en especial de las mujeres. Micrós (seudónimo del primero) caracterizó a la empleada doméstica como la sirviente fiel capaz de soportar vejaciones e incluso groserías por parte de sus empleadores o patrones. Fe el caso de Romana, "vieja fea y reumática" que pese a los malos tratos ofrece una "sumisión de perro golpeado" y que aunque era una vieja enferma aún se podía aprovechar de ella e incluso culparle "de cualquier pérdida" (Campo, 2014: 149-151). Gutiérrez Nájera se mofó de las ancianas que por permanecer atadas a su mundo y a sus tradiciones fueron incapaces de darse cuenta que el mundo avanzó hasta entrar en la época del progreso (Nájera, 2001: 325-328).

Por otro lado, en la correspondencia que cerca de los 43 años Laura Méndez de Cuenca, entabló con Enrique Olavarría y Ferrari le hacia saber que a esa edad ya comenzaba "a recibir las caricias de la vejez bajo la forma de toda clase de achaques" (Méndez, 2006: 36). En la misma tesitura, a la edad de 43 años Luis G. Urbina plasmó en el poema ¡Qué noche tan azul! la idea de una vejez triste, enferma y negra que añora con ahínco a la juventud (Urbina, 1987: 14-17).

Como podemos observar, la literatura es una fuente cuya riqueza debe ser considerada para estudiar a la historia de la vejez no sólo en México sino a nivel internacional. Las descripciones que en ella nos encontramos, los matices que distinguen la narrativa de los escritores analizados y la distinción que imprimen desde una perspectiva de género, son herramientas fundamentales para enriquecer cualquier investigación.

\section{LA DISCUSIÓN EN EL AULA}

En este apartado comparto las experiencias que para algunos estudiantes ha implicado reflexionar sobre el tema del envejecimiento histórico y actual. Esta última parte está construida con base en algunas breves entrevistas que les realicé. Cabe mencionar que durante el último año he implementado en mis cursos la historia de la vejez como otra herramienta para explicar la historia de este país y de su sociedad con resultados positivos que se reflejan en el interés de los estudiantes pero también en la posterior discusión y en las preguntas que entre ellos surgen.

Cuando al inicio del curso les propongo estudiar el Porfiriato desde el enfoque económico, político, social o cultural, les parece normal y próximo a sus intereses, pero cuando les comento que hablaremos sobre vejez y envejecimiento, generalmente noto que no están familiarizados con el tema y que por lo tanto, no encuentran mayor utilidad en su análisis. 
Esto lo adjudico principalmente a dos factores. El primero se relaciona con su propia resistencia a envejecer. Es el caso de Jerôme, francés de 43 años, quien comentó que la vejez es una etapa de la vida que "me da miedo un poco" porque es "la edad última antes de la muerte", sin embargo, afirmó que le gustaría llegar a ella "solamente si estoy en buena condición"; en opinión de Stephanie, alemana de 24 años, ella sostiene que no le gustaría envejecer porque "uno tiene más fuerzas cuando uno es joven", aunque podría aceptar ser vieja "sólo si tengo un buen retiro"; para Tatiana, estudiante de origen ruso de 33 años, llegar a la vejez implica un "cambio hormonal y la gente se vuelve loca y enojona".

La segunda razón se vincula con el escaso o nulo conocimiento sobre el tema que han tenido durante sus distintos niveles escolares. Niklas, estudiante alemán de 20 años refiere no recordar que en sus clases hayan hablado sobre el envejecimiento aunque actualmente "los periódicos hablan sobre eso a menudo", de manera similar, Amondi, de 45 años recuerda que sólo le hablaron sobre viejos en leyendas kenianas, "nada serio".

Durante esa primer sesión, les explico que la historia de la vejez es de aparición muy reciente a nivel internacional y que en México se ha estudiado sólo tangencialmente, dado que a diferencia de los niños o los adultos, los viejos no han sido considerados como actores sociales sino que se les ha visto como una carga tanto para el Estado como para la familia.

A medida que las sesiones se desarrollan, los estudiantes se van involucrando cada vez más, sobre todo cuando se dan cuenta lo poco que en sus países se les ha comentado sobre envejecimiento de la población. Un elemento interesante llega cuando analizamos los distintos textos literarios y ellos notan que las palabras viejo, vieja, anciano y anciana aparecen con frecuencia acompañadas de una serie de adjetivos que también suelen ser empleados en la literatura de sus países de origen (vieja arrugada, anciano achacoso, anciano enfermo, entre otros). Una pregunta que con frecuencia me suelen realizar es: "¿y por qué ahora dicen adulto mayor?" Es ahí en donde hablamos sobre eufemismos, prejuicios, estereotipos y un concepto llamado viejismo que tiene que ver con la discriminación (positiva o negativa) a la persona vieja por el simple hecho de serlo (Mendoza-Núñez, 2008).

Por supuesto que no todas las opiniones sobre el envejecimiento y la vejez son negativas. Para Klaudy, eslovaca de 19 años, "la vejez es una parte importante de la vida, un tiempo para mirar nuestro pasado y evaluar nuestras vidas". Siguiendo esa misma idea, Michiko, estudiante de origen japonés, argumentó que dicha etapa significa "una experiencia de vida, tranquilidad y aconsejar a los más jóvenes". Ella no tuvo problemas para aceptar que quiere envejecer puesto que "la vida es un proceso y la vejez (como la muerte) son parte de ello". Esta idea es compartida por Dorian, inglés de 45 años quien 
refiere que el envejecimiento se vincula con el estado de salud de la persona y por Domingos, estudiante de 33 años procedente de Guinea Bissau quien argumentó que llegar a ser viejo no es cuestión de querer: "tengo que llegar, voy a llegar si Dios me permite, tengo ganas de envejecer porque como me enseñaron las tradiciones en mi país, un viejo es una biblioteca, una persona que tiene experiencia". Al igual que el resto de los estudiantes entrevistados, Domingos señaló que a lo largo de su formación académica no le hablaron sobre el tema:

aquí en este curso que tomé el profesor me habló del envejecimiento, este tema me gustó muchísimo también, nunca he tomado un curso donde las personas hablan sobre él, se habla de la salud pero nunca del envejecimiento, por eso me gustó muchísimo [...] Para mí empezar a hablar del envejecimiento, tomar este tema como una cosa muy importante, una persona tiene que ser tratado con cariño y respeto.

\section{CONCLUSIONES}

Es necesario discutir y reflexionar sobre el tema del envejecimiento y de todo lo que esto conlleva a distintos niveles. El estudio del tema ha recibido atención desde otras áreas (sociología, antropología, medicina, psicología, demografía, entre otras) pero ha sido escasa desde la perspectiva histórica. Debido a ello me parece fundamental realizar una reflexión histórica sobre la vejez y el envejecimiento en México para entender lo que representaron los viejos para la sociedad pero también para comprender de qué formas la sociedad se relacionó con las personas envejecidas.

La experiencia de compartir mi investigación de doctorado con los estudiantes del nivel Básico 4 en el CEPE ha sido grata y reconfortante debido a que en primer lugar, en todos los cursos que he impartido me han referido que hasta ese momento sus profesores no habían considerado el tema del envejecimiento como un perspectiva de análisis. Por otro lado, la discusión que se genera el intercambio de ideas entre los estudiantes, la comparación de modelos con sus respectivos países, y sobre todo, la reflexión sobre su propio proceso de envejecimiento son elementos que enriquecen la experiencia docente. 
BiBLIOGRAFÍA

AHSS, BP, EA, HP - Archivo Histórico de la Secretaría de Salud, Beneficencia Pública, Establecimientos de Asistencia, Hospicio de Pobres, leg. 8, exp. 14, 17 de febrero de 1880.

AJAP - Archivo de la Junta de Asistencia Privada, Copia simple de la escritura de protocolización. Estatutos. Testamento de la fundación del asilo "Matías Romero", leg. 1, exp. 099/26, Ciudad de México.

ALBA, Víctor (1992), Historia social de la vejez, España: Laertes.

Arrom, Silvia Marina (2000). "El Hospicio de Pobres, un experimento fracasado de control social" en Carlos Illades y Ariel Rodríguez Kuri [coomps.], Instituciones y ciudad. Ocho estudios históricos sobre la Ciudad de México, México: Ediciones ¡Uníos! (Sábado Distrito Federal)

(2011) Para contener al pueblo: el Hospicio de Pobres en la Ciudad de México (1774-1871). México: Publicaciones de la Casa Chata.

Barbosa Cruz, Mario (2008). El trabajo en las calles. Subsistencia y negociación política en la Ciudad de México a comienzos del siglo XX, México: El Colegio de México.

BeAuvorr, Simone (1983). La vejez. México: Hermes.

Cole, Thomas R., Winkler, Mary G. (1994). The Oxford Book of Aging. Reflections on the Journey of Life. Oxford. Oxford University Press.

Cole, Thomas R. (2006). The Journey of Life. A Cultural History of Ageing in America. Cambridge: Cambridge University Press.

García González, Francisco (2005). Vejez, envejecimiento y sociedad en España. Siglos XVI-XXI. Cuenca: Universidad de Castilla-La Mancha. Garza, James Alex (2008). El lado oscuro del Porfiriato. Sexo, crímenes y vicios de la Ciudad de México. México: Aguilar.

González Navarro, Moisés (1970). Historia Moderna de México. El Porfiriato. Vida social. México: Hermes.

HACKet FisCheR, David (2015). Growing Old in America. Oxford: Oxford University Press.

Junta de Asistencia Privada (1934). Memoria que consigna la actuación de la Junta de Beneficencia Privada en el Distrito Federal, durante el periodo comprendido entre el mes de septiembre de 1932 y el de noviembre de 1934, bajo la presidencia del señor Don José M. Tapia. México: Cultura. 
LoRENZo Río, María Dolores (2011) El Estado como benefactor. Los pobres y la asistencia pública en la Ciudad de México. 1877-1905. México: El Colegio de México.

(2012). Los indigentes ante la asistencia pública. Una estrategia para sobrevivir en la Ciudad de México, 1877-1905, Historia Mexicana LXII, pp: 195247.

(2013). “¿De quién son los pobres? La experiencia del Asilo particular de mendigos" en Alicia Salmerón y Fernando Aguayo [coords.] Instantáneas de la ciudad de México. Un álbum de 1883-1884. México: Instituto José María Luis Mora, pp. 400-428.

Mendoza-NúÑez, Víctor [et.al.] (2008). Viejismo: prejuicios y estereotipos de la vejez. México: Facultad de Estudios Superiores Zaragoza.

Minols, George (1987). Historia de la vejez. Madrid: Nerea.

Mitchell, Margaret. (1997) The Porfirian State and Public Beneficence: The Hospicio de Pobres of Mexico City 1877-1911. Tulane: Tulane University (tesis de doctorado), 1988.

GutiérRez Nájera, Manuel (2001). Obras completas (XII). Narrativa, II. Relatos (18771894). México: Universidad Nacional Autónoma de México.

Prieto, Guillermo (2005). Obras completas (II). Cuadros de costumbres 1. México: Consejo Nacional para la Cultura y las Artes.

Speckman GuerRa, Elisa (2002). Crimen y castigo. Legislación penal, interpretaciones de la criminalidad y administración de justicia (Ciudad de México, 1872-1910), México: El Colegio de México.

Thane, Pat (2000). Old Age in English History. Past Experiences, Present Issues. Oxford: Oxford University Press. (2005). A History of Old Age. Los Angeles: The Paul Getty Museum.

UrBINA, Luis G. (1987). Poesías completas. Tomo II. México: Porrúa 
